\title{
ISOMONODROMIC DEFORMATIONS OF LOGARITHMIC CONNECTIONS AND STABILITY
}

\author{
INDRANIL BISWAS, VIKTORIA HEU, AND JACQUES HURTUBISE
}

\begin{abstract}
Let $X_{0}$ be a compact connected Riemann surface of genus $g$ with $D_{0} \subset X_{0}$ an ordered subset of cardinality $n$, and let $E_{G}$ be a holomorphic principal $G$-bundle on $X_{0}$, where $G$ is a reductive affine algebraic group defined over $\mathbb{C}$, that is equipped with a logarithmic connection $\nabla_{0}$ with polar divisor $D_{0}$. Let $\left(\mathcal{E}_{G}, \nabla\right)$ be the universal isomonodromic deformation of $\left(E_{G}, \nabla_{0}\right)$ over the universal Teichmüller curve $(\mathcal{X}, \mathcal{D}) \longrightarrow$ Teich $_{g, n}$, where Teich $_{g, n}$ is the Teichmüller space for genus $g$ Riemann surfaces with $n$-marked points. We prove the following (see Section [5):

(1) Assume that $g \geq 2$ and $n=0$. Then there is a closed complex analytic subset $\mathcal{Y} \subset \operatorname{Teich}_{g, n}$, of codimension at least $g$, such that for any $t \in \operatorname{Teich}_{g, n} \backslash \mathcal{Y}$, the principal $G$-bundle $\left.\mathcal{E}_{G}\right|_{\mathcal{X}_{t}}$ is semistable, where $\mathcal{X}_{t}$ is the compact Riemann surface over $t$.

(2) Assume that $g \geq 1$, and if $g=1$, then $n>0$. Also, assume that the monodromy representation for $\nabla_{0}$ does not factor through some proper parabolic subgroup of $G$. Then there is a closed complex analytic subset $\mathcal{Y}^{\prime} \subset$ Teich $_{g, n}$, of codimension at least $g$, such that for any $t \in \operatorname{Teich}_{g, n} \backslash \mathcal{Y}^{\prime}$, the principal $G$-bundle $\left.\mathcal{E}_{G}\right|_{\mathcal{X}_{t}}$ is semistable.

(3) Assume that $g \geq 2$. Assume that the monodromy representation for $\nabla_{0}$ does not factor through some proper parabolic subgroup of $G$. Then there is a closed complex analytic subset $\mathcal{Y}^{\prime \prime} \subset \operatorname{Teich}_{g, n}$, of codimension at least $g-1$, such that for any $t \in \operatorname{Teich}_{g, n} \backslash \mathcal{Y}^{\prime}$, the principal $G$-bundle $\left.\mathcal{E}_{G}\right|_{\mathcal{X}_{t}}$ is stable.

In $\mathrm{He} 1$, the second-named author proved the above results for $G=\operatorname{GL}(2, \mathbb{C})$.
\end{abstract}

\section{INTRODUCTION}

Take any quadruple of the form $(E \longrightarrow X, D, \nabla)$, where $E$ is a holomorphic vector bundle over a smooth connected complex variety $X$, and $\nabla$ is an integrable logarithmic connection on $E$ singular over a simple normal crossing divisor $D \subset X$. The monodromy functor associates to it a representation $\rho_{\nabla}: \pi_{1}\left(X \backslash D, x_{0}\right) \longrightarrow \operatorname{GL}\left(E_{x_{0}}\right)$, where $x_{0} \in X \backslash D$. Altering the connection by a holomorphic automorphism $A$ of $E$ leads to a representation conjugated by $A\left(x_{0}\right)$. The monodromy functor produces an equivalence between the category of logarithmic connections $(E, \nabla)$ on $(X, D)$ such that the real parts of the residues lie in $[0,1)$ and the category of equivalence classes of linear representations of $\pi_{1}\left(X \backslash D, x_{0}\right)$. Given a monodromy representation $\rho$, one can consider the set of all logarithmic connections $(E \longrightarrow X, D, \nabla)$ (with no condition on the residues) up to holomorphic isomorphisms that produce the same monodromy representation $\rho=\rho_{\nabla}$ up to conjugation. All these connections are conjugated to each other by meromorphic gauge transformations with possible poles over $D$ (see for example [Sa]).

2010 Mathematics Subject Classification. 14H60, 53B15.

Key words and phrases. Logarithmic connection, isomonodromic deformation, stability, principal bundle. 
The classical Riemann-Hilbert problem can be formulated as follows:

Given a representation $\rho: \pi_{1}\left(\mathbb{P}_{\mathbb{C}}^{1} \backslash D_{0}, x\right) \longrightarrow \mathrm{GL}(r, \mathbb{C})$, is there a logarithmic connection $\left(E \longrightarrow \mathbb{P}_{\mathbb{C}}^{1}, D_{0}, \nabla\right)$ such that $\rho=\rho_{\nabla}$ and $E$ is holomorphically trivial?

The answer to this problem is

(1) positive if rank $r=2[\mathrm{Pl}],[\mathrm{De}$,

(2) negative in general $(r \geq 3)[\mathrm{AB}$,

(3) positive if $\rho$ is irreducible [Bol1], [Ko].

On the other hand, the fundamental group $\pi_{1}\left(\mathbb{P}_{\mathbb{C}}^{1} \backslash D_{0}, x\right)$ depends only on the topological and not the complex structure of $\mathbb{P}_{\mathbb{C}}^{1} \backslash D_{0}$. So given an integrable connection on $\mathbb{P}_{\mathbb{C}}^{1} \backslash$ $D_{0}$, one can consider variations of the complex structure without changing the monodromy representation. More precisely, consider the Teichmüller space Teich ${ }_{0, n}$ of the $n$-pointed Riemann sphere together with the corresponding universal Teichmüller curve

$$
\tau:\left(\mathcal{X}=\mathbb{P}_{\mathbb{C}}^{1} \times \text { Teich }_{0, n}, \mathcal{D}\right) \longrightarrow \text { Teich }_{0, n}
$$

where $n=\operatorname{degree}\left(D_{0}\right)$. Since Teich ${ }_{0, n}$ is contractible, the inclusion

$$
\left(\mathbb{P}_{\mathbb{C}}^{1}, D_{t}\right):=\tau^{-1}(t) \hookrightarrow(\mathcal{X}, \mathcal{D}), \quad t \in \text { Teich }_{0, n},
$$

induces an isomorphism $\pi_{1}\left(\mathbb{P}_{\mathbb{C}}^{1} \backslash D_{t}, x_{t}\right) \simeq \pi_{1}\left(\mathcal{X} \backslash \mathcal{D}, x_{t}\right)$. Hence by the Riemann-Hilbert correspondence, we can associate to any logarithmic connection $\left(E_{0}, \nabla_{0}\right)$ on $\mathbb{P}_{\mathbb{C}}^{1}$, with polar divisor $D_{0}$, its universal isomonodromic deformation: a flat logarithmic connection $(\mathcal{E}, \nabla)$ over $\mathcal{X}$ with polar divisor $\mathcal{D}$ that extends the connection $\left(E_{0}, \nabla_{0}\right)$. The conjugacy class of the monodromy representation for $\left.\nabla\right|_{\tau^{-1}(t)}$ does not change as $t$ moves over Teich ${ }_{0, n}$ (see for example [He2]).

We are led to another Riemann-Hilbert problem:

Given a logarithmic connection $\left(E_{0}, \nabla_{0}\right)$ on $\mathbb{P}_{\mathbb{C}}^{1}$ with polar divisor $D_{0}$ of degree $n$, is there a point $t \in$ Teich $_{0, n}$ such that the holomorphic vector bundle $E_{t}=\left.\mathcal{E}\right|_{\mathbb{P}_{\mathbb{C}}^{1} \times\{t\}}$ underlying the universal isomonodromic deformation $(\mathcal{E}, \nabla)$ is trivial?

A partial answer to this question is given by the following theorem of Bolibruch:

Theorem 1.1 ([Bol2] $)$. Let $\left(E_{0}, \nabla_{0}\right)$ be an irreducible trace-free logarithmic rank two connection with $n \geq 4$ poles on $\mathbb{P}_{\mathbb{C}}^{1}$ such that each singularity is resonant. There is a proper closed complex analytic subset $\mathcal{Y} \subset$ Teich $_{0, n}$ such that for all $t \in \operatorname{Teich}_{0, n} \backslash \mathcal{Y}$, the holomorphic vector bundle $E_{t}=\left.\mathcal{E}\right|_{\mathbb{P}_{\mathbb{C}}^{1} \times\{t\}}$ underlying the universal isomonodromic deformation $(\mathcal{E}, \nabla)$ of $\left(E_{0}, \nabla_{0}\right)$ is trivial.

It should be mentioned that the condition in Theorem[1.1, that each singularity is resonant, can actually be removed [He1].

From the Birkhoff-Grothendieck classification of holomorphic vector bundles on $\mathbb{P}_{\mathbb{C}}^{1}$ it follows immediately that the only semistable holomorphic vector bundle of degree zero and rank $r$ on $\mathbb{P}_{\mathbb{C}}^{1}$ is the trivial bundle $\mathcal{O}_{\mathbb{P}_{\mathbb{C}}^{1}}^{\oplus r}$. This leads to the following more general question: 
Given a representation $\rho: \pi_{1}(X \backslash D, x) \longrightarrow \mathrm{GL}_{r}(\mathbb{C})$, where $X$ is a compact connected Riemann surface, is there a logarithmic connection $(E \longrightarrow X, D, \nabla)$ such that $\rho=\rho_{\nabla}$ and $E$ is semistable of degree zero?

The answer to this problem is

(1) negative in general $[\mathrm{EH}$,

(2) positive if $\rho$ is irreducible [EV].

Let $\tau:(\mathcal{X}, \mathcal{D}) \longrightarrow$ Teich $_{g, n}$ be the universal Teichmüller curve. In view of the above, it is natural to ask the following:

Given a logarithmic connection $\left(E_{0}, \nabla_{0}\right)$, with polar divisor $D_{0}$ of degree $n$ on a compact connected Riemann surface $X_{0}$ of genus $g$, is there an element $t \in$ Teich $_{g, n}$ such that the holomorphic vector bundle $E_{t}=\left.\mathcal{E}\right|_{\mathcal{X}_{t}} \longrightarrow \mathcal{X}_{t}=\tau^{-1}(t)$ underlying the universal isomonodromic deformation $(\mathcal{E}, \nabla)$ of $\left(E_{0}, \nabla_{0}\right)$ is semistable?

Note that we necessarily have $\operatorname{degree}\left(E_{t}\right)=\operatorname{degree}\left(E_{0}\right)$. Again, Theorem 1.1 can be generalized as follows.

Theorem 1.2 ([He1]). Let $\left(E_{0}, \nabla_{0}\right)$ be an irreducible logarithmic rank two connection with polar divisor $D_{0}$ of degree $n$ on a compact connected Riemann surface $X_{0}$ of genus $g$ such that $3 g-3+n>0$. Consider its universal isomonodromic deformation $(\mathcal{E}, \nabla)$ over $\tau$ : $(\mathcal{X}, \mathcal{D}) \longrightarrow$ Teich $_{g, n}$. There is a closed complex analytic subset $\mathcal{Y} \subset$ Teich $_{g, n}$ (respectively, $\mathcal{Y}_{s} \subset \operatorname{Teich}_{g, n}$ ) of codimension at least $g$ (respectively, $g-1$ ) such that for any $t \in \operatorname{Teich}_{g, n} \backslash$ $\mathcal{Y}$, the vector bundle $E_{t}=\left.\mathcal{E}\right|_{\mathcal{X}_{t}}$, where $\left(\mathcal{X}_{t}, D_{t}\right)=\tau^{-1}(t)$, is semistable (respectively, stable).

Our aim here is to prove an analog of Theorem 1.2 in the more general context of logarithmic connections on principal $G$-bundles over a compact connected Riemann surface (see Boa] for logarithmic connections on principal $G$-bundles).

Let $X_{0}$ be a compact connected Riemann surface of genus $g$, and let $D_{0} \subset X_{0}$ be an ordered subset of it of cardinality $n$. Let $G$ be a reductive affine algebraic group defined over C. Let $E_{G}$ be a holomorphic principal $G$-bundle on $X_{0}$ and $\nabla_{0}$ a logarithmic connection on $E_{G}$ with polar divisor $D_{0}$. Let $\left(\mathcal{E}_{G}, \nabla\right)$ be the universal isomonodromic deformation of $\left(E_{G}, \nabla_{0}\right)$ over the universal Teichmüller curve $\tau:(\mathcal{X}, \mathcal{D}) \longrightarrow$ Teich $_{g, n}$. For any point $t \in$ Teich $_{g, n}$, the restriction $\left.\mathcal{E}_{G}\right|_{\tau^{-1}(t)} \longrightarrow \mathcal{X}_{t}:=\tau^{-1}(t)$ will be denoted by $\mathcal{E}_{G}^{t}$.

We prove the following (see Section 5 ):

\section{Theorem 1.3.}

(1) Assume that $g \geq 2$ and $n=0$. Then there is a closed complex analytic subset $\mathcal{Y} \subset$ Teich $_{g, n}$ of codimension at least $g$ such that for any $t \in \operatorname{Teich}_{g, n} \backslash \mathcal{Y}$, the holomorphic principal $G$-bundle $\mathcal{E}_{G}^{t} \longrightarrow \mathcal{X}_{t}$ is semistable.

(2) Assume that $g \geq 1$, and if $g=1$, then $n>0$. Also, assume that the monodromy representation for $\nabla_{0}$ does not factor through some proper parabolic subgroup of $G$. Then there is a closed complex analytic subset $\mathcal{Y}^{\prime} \subset$ Teich $_{g, n}$ of codimension at least $g$ such that for any $t \in \operatorname{Teich}_{g, n} \backslash \mathcal{Y}^{\prime}$, the holomorphic principal $G$-bundle $\mathcal{E}_{G}^{t}$ is semistable. 
(3) Assume that $g \geq 2$. Assume that the monodromy representation for $\nabla_{0}$ does not factor through some proper parabolic subgroup of $G$. Then there is a closed complex analytic subset $\mathcal{Y}^{\prime \prime} \subset$ Teich $_{g, n}$ of codimension at least $g-1$ such that for any $t \in$ Teich $_{g, n} \backslash \mathcal{Y}^{\prime}$, the holomorphic principal $G$-bundle $\mathcal{E}_{G}^{t}$ is stable.

It is known that if a holomorphic principal $G$-bundle $E_{G}$ over a complex elliptic curve admits a holomorphic connection, then $E_{G}$ is semistable. Therefore, a stronger version of Theorem 1.3. (1) is valid when $g=1$.

\section{INFINITESIMAL DEFORMATIONS}

We first recall some classical results in deformation theory, and in the process setting up our notation.

2.1. Deformations of a $n$-pointed curve. Let $X_{0}$ be an irreducible smooth complex projective curve of genus $g$, with $g>0$, and let

$$
D_{0}:=\left\{x_{1}, \cdots, x_{n}\right\} \subset X_{0}
$$

be an ordered subset of cardinality $n$ (it may be zero). We assume that $n>0$ if $g=1$. This condition implies that the pair $\left(X_{0}, D_{0}\right)$ does not have any infinitesimal automorphism, equivalently, the automorphism group of $\left(X_{0}, D_{0}\right)$ is finite.

Let $B:=\operatorname{Spec}\left(\mathbb{C}[\epsilon] / \epsilon^{2}\right)$ be the spectrum of the local ring. An infinitesimal deformation of $\left(X_{0}, D_{0}\right)$ is given by a quadruple

$$
(\mathcal{X}, q, \mathcal{D}, f)
$$

where

- $q: \mathcal{X} \longrightarrow B$ is a smooth proper holomorphic morphism of relative dimension one,

- $\mathcal{D}=\left(\mathcal{D}_{1}, \cdots, \mathcal{D}_{n}\right)$ is a collection of $n$ ordered disjoint sections of $q$, and

- $f: X_{0} \longrightarrow \mathcal{X}$ is a holomorphic morphism such that

$$
f\left(X_{0}\right) \subset \mathcal{X}_{0}:=q^{-1}(0) \text { with } f\left(x_{i}\right)=\mathcal{D}_{i}(0) \quad \forall 1 \leq i \leq n,
$$

and the morphism $X_{0} \stackrel{f}{\longrightarrow} \mathcal{X}_{0}$ is an isomorphism.

The divisor $\sum_{i=1}^{n} \mathcal{D}_{i}(B)$ on $\mathcal{X}$ will also be denoted by $\mathcal{D}$. For a vector bundle $\mathcal{V}$ on $\mathcal{X}$, the vector bundle $\mathcal{V} \otimes_{\mathcal{O}_{\mathcal{X}}} \mathcal{O}_{\mathcal{X}}(-\mathcal{D})$ will be denoted by $\mathcal{V}(-\mathcal{D})$.

The differential of $f$

$$
\mathrm{d} f: \mathrm{T} X_{0} \longrightarrow f^{*} \mathrm{~T} \mathcal{X}
$$

produces a homomorphism

$$
\mathrm{T} X_{0}\left(-D_{0}\right):=\mathrm{T} X_{0} \otimes_{\mathcal{O}_{X_{0}}} \mathcal{O}_{X_{0}}\left(-D_{0}\right) \longrightarrow f^{*} \mathrm{~T} \mathcal{X}(-\mathcal{D})
$$

which will also be denoted by $\mathrm{d} f$. Consider the following short exact sequence of coherent sheaves on $X_{0}$ :

$$
0 \longrightarrow \mathrm{T} X_{0}\left(-D_{0}\right) \stackrel{\mathrm{d} f}{\longrightarrow} f^{*} \mathrm{~T} \mathcal{X}(-\mathcal{D}) \stackrel{h}{\longrightarrow} \mathcal{O}_{X_{0}} \longrightarrow 0
$$


note that the normal bundle to $\mathcal{X}_{0} \subset \mathcal{X}$ is the pullback of $\mathrm{T}_{0} B$ by $\left.q\right|_{\mathcal{X}_{0}}$, and hence this normal bundle is identified with $\mathcal{O}_{X_{0}}$. Consider the connecting homomorphism

$$
\mathbb{C}=\mathrm{H}^{0}\left(X_{0}, \mathcal{O}_{X_{0}}\right) \stackrel{\phi}{\longrightarrow} \mathrm{H}^{1}\left(X_{0}, \mathrm{~T} X_{0}\left(-D_{0}\right)\right)
$$

in the long exact sequence of cohomologies associated to the short exact sequence in (2.2). Let $1_{X_{0}}$ denote the constant function 1 on $X_{0}$. The cohomology element

$$
\phi\left(1_{X_{0}}\right) \in \mathrm{H}^{1}\left(X_{0}, \mathrm{~T} X_{0}\left(-D_{0}\right)\right),
$$

where $\phi$ is the homomorphism in (2.3), is the Kodaira-Spencer infinitesimal deformation class for the family in (2.1). If this infinitesimal deformation class is zero, then the family $(\mathcal{X}, \mathcal{D}) \longrightarrow B$ is isomorphic to the trivial family $\left(X_{0} \times B, D_{0} \times B\right) \longrightarrow B$.

2.2. Deformations of a curve together with a principal bundle. Take $\left(X_{0}, D_{0}\right)$ as before. Let $G$ be a connected reductive affine algebraic group defined over $\mathbb{C}$. The Lie algebra of $G$ will be denoted by $\mathfrak{g}$. Let

$$
p: E_{G} \longrightarrow X_{0}
$$

be a holomorphic principal $G$-bundle on $X_{0}$. The infinitesimal deformations of the triple

$$
\left(X_{0}, D_{0}, E_{G}\right)
$$

are guided by the Atiyah bundle $\operatorname{At}\left(E_{G}\right) \longrightarrow X_{0}$, the construction of which we shall briefly recall (see [At] for a more detailed exposition). Consider the direct image $p_{*} \mathrm{~T} E_{G} \longrightarrow X_{0}$, where $\mathrm{T} E_{G}$ is the holomorphic tangent bundle of $E_{G}$, and $p$ is the projection in (2.5). It is a quasicoherent sheaf equipped with an action of $G$ given by the action of $G$ on $E_{G}$. The invariant part

$$
\operatorname{At}\left(E_{G}\right):=\left(p_{*} \mathrm{~T} E_{G}\right)^{G} \subset\left(p_{*} \mathrm{~T} E_{G}\right)
$$

is a vector bundle on $X_{0}$ of $\operatorname{rank} 1+\operatorname{dim} G$ which is known as the Atiyah bundle of $E_{G}$. Consequently, we have $\operatorname{At}\left(E_{G}\right)=\left(T E_{G}\right) / G$. Let

$$
\operatorname{ad}\left(E_{G}\right):=E_{G} \times^{G} \mathfrak{g} \longrightarrow X_{0}
$$

be the adjoint vector bundle associated to $E_{G}$ for the adjoint action of $G$ on its Lie algebra $\mathfrak{g}$. So the fibers of $\operatorname{ad}\left(E_{G}\right)$ are Lie algebras isomorphic to $\mathfrak{g}$. Let

$$
\mathrm{d} p: \mathrm{TE}_{G} \longrightarrow p^{*} \mathrm{~T} X_{0}
$$

be the differential of the map $p$ in (2.5). Being $G$-equivariant it produces a homomorphism $\operatorname{At}\left(E_{G}\right) \longrightarrow \mathrm{T} X_{0}$ which will also be denoted by $\mathrm{d} p$. Now, the action of $G$ on $E_{G}$ produces an isomorphism $E_{G} \times \mathfrak{g} \longrightarrow \operatorname{kernel}(\mathrm{d} p)$. Therefore, we have $\operatorname{kernel}(\mathrm{d} p) / G=\operatorname{ad}\left(E_{G}\right)$. In other words, the above isomorphism $E_{G} \times \mathfrak{g} \longrightarrow \operatorname{kernel}(\mathrm{d} p)$ descends to an isomorphism

$$
\operatorname{ad}\left(E_{G}\right) \stackrel{\sim}{\longrightarrow}\left(p_{*}(\operatorname{kernel}(\mathrm{d} p))\right)^{G}
$$

that preserves the Lie algebra structure on the fibers of the two vector bundles (the Lie algebra structure on the fibers of $\left(p_{*}(\operatorname{kernel}(\mathrm{d} p))\right)^{G}$ is given by the Lie bracket of $G$-invariant vertical vector fields). Therefore, $\operatorname{At}\left(E_{G}\right)$ fits in the following short exact sequence of vector bundles on $X_{0}$

$$
0 \longrightarrow \operatorname{ad}\left(E_{G}\right) \longrightarrow \operatorname{At}\left(E_{G}\right) \stackrel{\mathrm{d} p}{\longrightarrow} \mathrm{T} X_{0} \longrightarrow 0
$$


which is known as the Atiyah exact sequence for $E_{G}$. The logarithmic Atiyah bundle $\operatorname{At}\left(E_{G}, D_{0}\right)$ is defined by

$$
\operatorname{At}\left(E_{G}, D_{0}\right):=(\mathrm{d} p)^{-1}\left(\mathrm{~T} X_{0}\left(-D_{0}\right)\right) \subset \operatorname{At}\left(E_{G}\right) .
$$

From (2.7) we have the short exact sequence of vector bundles on $X_{0}$

$$
0 \longrightarrow \operatorname{ad}\left(E_{G}\right) \longrightarrow \operatorname{At}\left(E_{G}, D_{0}\right) \stackrel{\sigma}{\longrightarrow} \mathrm{T} X_{0}\left(-D_{0}\right) \longrightarrow 0,
$$

which is called the logarithmic Atiyah exact sequence. The above homomorphism $\sigma$ is the restriction of $\mathrm{d} p$ to $\operatorname{At}\left(E_{G}, D_{0}\right) \subset \operatorname{At}\left(E_{G}\right)$.

An infinitesimal deformation of the triple $\left(X_{0}, D_{0}, E_{G}\right)$ in (2.6) is a 6-tuple

$$
\left(\mathcal{X}, q, \mathcal{D}, f, \mathcal{E}_{G}, \psi\right)
$$

where

- $(\mathcal{X}, q, \mathcal{D}, f)$ in an infinitesimal deformation of the $n$-pointed curve $\left(X_{0}, D_{0}\right)$ as in $(2.1)$

- $\mathcal{E}_{G} \longrightarrow \mathcal{X}$ is a holomorphic principal $G$-bundle, and

- $\psi$ is a holomorphic isomorphism

$$
\psi: E_{G} \longrightarrow f^{*} \mathcal{E}_{G}
$$

of principal $G$-bundles.

The logarithmic Atiyah bundle

$$
\operatorname{At}\left(\mathcal{E}_{G}, \mathcal{D}\right) \longrightarrow \mathcal{X}
$$

for $\left(\mathcal{E}_{G}, \mathcal{D}\right)$ is the inverse image, in $\operatorname{At}\left(\mathcal{E}_{G}\right)$, of the subsheaf $\left.\mathrm{T} \mathcal{X}(-\mathcal{D})\right) \subset \mathrm{T} \mathcal{X}$. We have the following short exact sequence of sheaves on $X_{0}$ :

$$
0 \longrightarrow \operatorname{At}\left(E_{G}, D_{0}\right) \longrightarrow f^{*} \operatorname{At}\left(\mathcal{E}_{G}, \mathcal{D}\right) \longrightarrow \mathcal{O}_{X_{0}} \longrightarrow 0
$$

given by $\psi$ in (2.10). Let

$$
\mathbb{C}=\mathrm{H}^{0}\left(X_{0}, \mathcal{O}_{X_{0}}\right) \stackrel{\widetilde{\phi}}{\longrightarrow} \mathrm{H}^{1}\left(X_{0}, \operatorname{At}\left(E_{G}, D_{0}\right)\right)
$$

be the connecting homomorphism in the long exact sequence of cohomologies associated to (2.11). The cohomology element

$$
\widetilde{\phi}\left(1_{X_{0}}\right) \in \mathrm{H}^{1}\left(X_{0}, \operatorname{At}\left(E_{G}, D_{0}\right)\right)
$$

is the cohomology class of the infinitesimal deformation of the triple $\left(X_{0}, D_{0}, E_{G}\right)$ given by (2.9). Let

$$
\sigma_{*}: \mathrm{H}^{1}\left(X_{0}, \operatorname{At}\left(E_{G}, D_{0}\right)\right) \longrightarrow \mathrm{H}^{1}\left(X_{0}, \mathrm{~T} X_{0}\left(-D_{0}\right)\right)
$$

be the homomorphism given by the projection $\sigma$ in (2.8). From the commutativity of the diagram

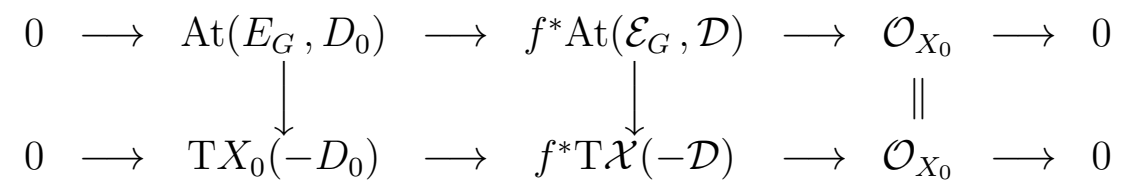

where the top and bottom rows are as in (2.11) and (2.2) respectively, it follows that

$$
\sigma_{*}\left(\widetilde{\phi}\left(1_{X_{0}}\right)\right)=\phi\left(1_{X_{0}}\right)
$$


where $\widetilde{\phi}\left(1_{X_{0}}\right)$ and $\phi\left(1_{X_{0}}\right)$ are constructed in (2.12) and (2.4) respectively. We note that $\sigma_{*}$ is the forgetful map that sends an infinitesimal deformation of $\left(X_{0}, D_{0}, E_{G}\right)$ to the underlying infinitesimal deformation of $\left(X_{0}, D_{0}\right)$ forgetting the principal $G$-bundle.

\section{Obstruction to extension of a Reduction of structure group to $P$}

Given a holomorphic reduction of structure group of $E_{G}$ to a parabolic subgroup of $G$, our aim in this section is to compute the obstruction for this reduction to extend to a reduction of an infinitesimal deformation $\mathcal{E}_{G} \longrightarrow \mathcal{X}$ as in (2.9) (see the paragraph after the proof of Lemma 3.1).

A parabolic subgroup of $G$ is a connected Zariski closed subgroup $P$ such that $G / P$ is a complete variety. Fix a parabolic subgroup $P \subset G$. The Lie algebra of $P$ will be denoted by $\mathfrak{p}$. As before, $E_{G}$ is a holomorphic principal $G$-bundle on $X_{0}$. Let

$$
E_{P} \subset E_{G}
$$

be a holomorphic reduction of structure group of $E_{G}$ to the subgroup $P \subset G$. Let

$$
\operatorname{ad}\left(E_{P}\right):=E_{P} \times^{P} \mathfrak{p} \longrightarrow X_{0}
$$

be the adjoint vector bundle associated to $E_{P}$ for the adjoint action of $P$ on its Lie algebra $\mathfrak{p}$. The vector bundle over $X_{0}$ associated to the principal $P$-bundle $E_{P}$ for the adjoint action of $P$ on the quotient $\mathfrak{g} / \mathfrak{p}$ will be denoted by $E_{P}(\mathfrak{g} / \mathfrak{p})$. So, $E_{P}(\mathfrak{g} / \mathfrak{p})=\operatorname{ad}\left(E_{G}\right) / \operatorname{ad}\left(E_{P}\right)$. The logarithmic Atiyah bundle for $\left(E_{P}, D_{0}\right)$ will be denoted by $\operatorname{At}\left(E_{P}, D_{0}\right)$. We have

$$
\operatorname{ad}\left(E_{P}\right) \subset \operatorname{ad}\left(E_{G}\right) \text { and } \operatorname{At}\left(E_{P}, D_{0}\right) \subset \operatorname{At}\left(E_{G}, D_{0}\right)
$$

both the quotient bundles above are identified with $E_{P}(\mathfrak{g} / \mathfrak{p})$. In other words, we have the following commutative diagram of vector bundles on $X_{0}$

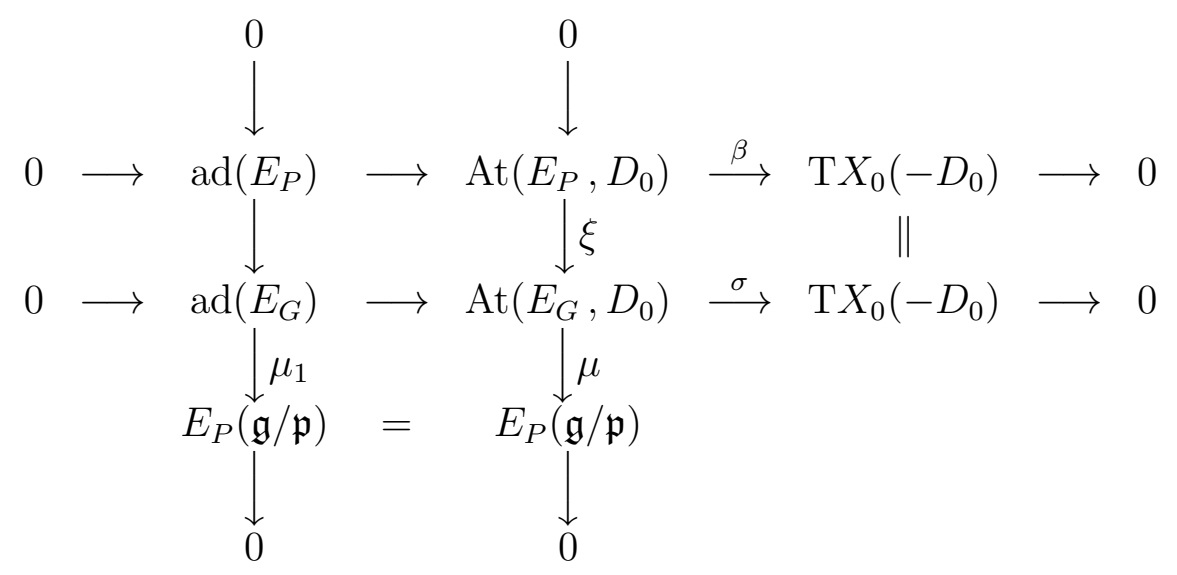

where $\sigma$ is the homomorphism in (2.8). Let

$$
\widetilde{\xi}: \mathrm{H}^{1}\left(X_{0}, \operatorname{At}\left(E_{P}, D_{0}\right)\right) \longrightarrow \mathrm{H}^{1}\left(X_{0}, \operatorname{At}\left(E_{G}, D_{0}\right)\right)
$$

be the homomorphism induced by the canonical injection $\xi$ in (3.2).

Take any $\left(\mathcal{X}, q, \mathcal{D}, f, \mathcal{E}_{G}, \psi\right)$ as in (2.9). Assume that the reduction $E_{P} \subset E_{G}$ in (3.1) extends to a holomorphic reduction of structure group

$$
\mathcal{E}_{P} \subset \mathcal{E}_{G}
$$


to $P \subset G$ on $\mathcal{X}$. Consider the short exact sequence on $X_{0}$

$$
0 \longrightarrow \operatorname{At}\left(E_{P}, D_{0}\right) \longrightarrow f^{*} \operatorname{At}\left(\mathcal{E}_{P}, \mathcal{D}\right) \longrightarrow \mathcal{O}_{X_{0}} \longrightarrow 0
$$

where $\operatorname{At}\left(\mathcal{E}_{P}, \mathcal{D}\right) \longrightarrow \mathcal{X}$ is the logarithmic Atiyah bundle associated to the principal $P$ bundle $\mathcal{E}_{P}$, and $f$ is the map in (2.1). Let

$$
\theta \in \mathrm{H}^{1}\left(X_{0}, \operatorname{At}\left(E_{P}, D_{0}\right)\right)
$$

be the image of the constant function $1_{X_{0}}$ by the homomorphism

$$
\mathrm{H}^{0}\left(X_{0}, \mathcal{O}_{X_{0}}\right) \longrightarrow \mathrm{H}^{1}\left(X_{0}, \operatorname{At}\left(E_{P}, D_{0}\right)\right)
$$

in the long exact sequence of cohomologies associated to (3.4).

Lemma 3.1. The cohomology class $\theta$ in (3.5) satisfies the equation

$$
\widetilde{\xi}(\theta)=\widetilde{\phi}\left(1_{X_{0}}\right),
$$

where $\widetilde{\xi}$ and $\widetilde{\phi}\left(1_{X_{0}}\right)$ are constructed in (3.3) and (2.12) respectively.

Proof. Consider the commutative diagram of vector bundles on $X_{0}$

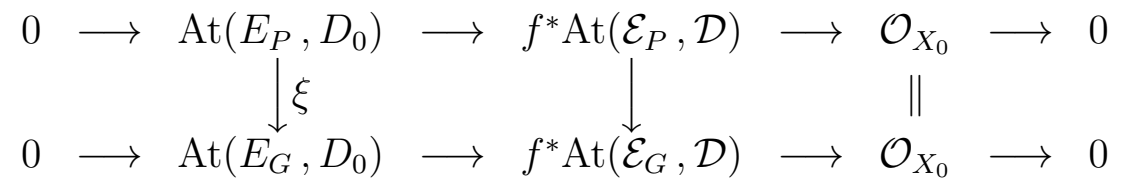

where the top and bottom rows are as in (3.4) and (2.11) respectively, and $\xi$ is the homomorphism in (3.2); the above homomorphism $f^{*} \operatorname{At}\left(\mathcal{E}_{P}, \mathcal{D}\right) \longrightarrow f^{*} \operatorname{At}\left(\mathcal{E}_{G}, \mathcal{D}\right)$ is the pullback of the natural homomorphism $\operatorname{At}\left(\mathcal{E}_{P}, \mathcal{D}\right) \longrightarrow \operatorname{At}\left(\mathcal{E}_{G}, \mathcal{D}\right)$. In view of (3.6), the lemma follows by comparing the constructions of $\theta$ and $\widetilde{\phi}\left(1_{X_{0}}\right)$.

Consider the homomorphism $\mu_{*}: \mathrm{H}^{1}\left(X_{0}, \operatorname{At}\left(E_{G}, D_{0}\right)\right) \longrightarrow \mathrm{H}^{1}\left(X_{0}, E_{P}(\mathfrak{g} / \mathfrak{p})\right)$ induced by the homomorphism $\mu$ in (3.2). From Lemma 3.1 we conclude that $\mu_{*}\left(\widetilde{\phi}\left(1_{X_{0}}\right)\right)=0$; to see this consider the long exact sequence of cohomologies associated to the right vertical exact sequence in (3.2). Therefore, $\mu_{*}\left(\widetilde{\phi}\left(1_{X_{0}}\right)\right)$ is an obstruction for the reduction $E_{P} \subset E_{G}$ to extend to a reduction of $\mathcal{E}_{G}$ to $P$.

\section{LOGARITHMiC CONNECTIONS AND THE SECOND FUNDAMENTAL FORM}

In this section we characterize those infinitesimal deformations of the principal bundle $E_{G}$ on the $n$-pointed curve that arise from the isomonodromic deformations.

4.1. Canonical extension of a logarithmic connection. As before, let $p: E_{G} \longrightarrow X_{0}$ be a holomorphic principal $G$-bundle. A logarithmic connection on $E_{G}$ with polar divisor $D_{0}$ is a holomorphic splitting of the logarithmic Atiyah exact sequence in (2.8). In other words, a logarithmic connection is a homomorphism

$$
\delta: \operatorname{T} X_{0}\left(-D_{0}\right) \longrightarrow \operatorname{At}\left(E_{G}, D_{0}\right)
$$

such that $\sigma \circ \delta=\operatorname{Id}_{\mathrm{T} X_{0}\left(-D_{0}\right)}$, where $\sigma$ is the homomorphism in (2.8). Note that given such a $\delta$, there is a unique homomorphism

$$
\delta^{\prime \prime}: \operatorname{At}\left(E_{G}, D_{0}\right) \longrightarrow \operatorname{ad}\left(E_{G}\right)
$$


such that $\delta^{\prime \prime} \circ \delta=0$, and the composition

$$
\operatorname{ad}\left(E_{G}\right) \hookrightarrow \operatorname{At}\left(E_{G}, D_{0}\right) \stackrel{\delta^{\prime \prime}}{\longrightarrow} \operatorname{ad}\left(E_{G}\right)
$$

(see (2.8)) is the identity map of $\operatorname{ad}\left(E_{G}\right)$. As there are no nonzero $(2,0)$-forms on $X_{0}$, all logarithmic connections on $E_{G}$ are automatically integrable.

At the level of first order infinitesimal deformations, given a principal $G$-bundle

$$
\mathcal{E}_{G} \longrightarrow \mathcal{X} \stackrel{q}{\longrightarrow} B
$$

a logarithmic connection on $\mathcal{E}_{G}$ with polar divisor $\mathcal{D}$ is a homomorphism $\operatorname{At}\left(\mathcal{E}_{G}, \mathcal{D}\right) \longrightarrow$ $\operatorname{ad}\left(\mathcal{E}_{G}\right)$ that splits the logarithmic Atiyah exact sequence for $\mathcal{E}_{G}$. We note that a connection on $\mathcal{E}_{G}$ need not be integrable, as we have added an (infinitesimal) extra dimension. However, the Riemann-Hilbert correspondence for principal $G$-bundles yields the following:

Lemma 4.1. Let $(\mathcal{X}, q, \mathcal{D}, f)$ be an infinitesimal deformation of $\left(X_{0}, D_{0}\right)$ as in (2.1). Let $\delta$ be a logarithmic connection on a holomorphic principal $G$-bundle $E_{G}$ on $X_{0}$ with polar divisor $D_{0}$. Then there exists a unique pair $\left(\mathcal{E}_{G}, \nabla\right)$, where

- $\mathcal{E}_{G}$ is a holomorphic principal $G$-bundle on $\mathcal{X}$, and

- $\nabla$ is an integrable logarithmic connection on $\mathcal{E}_{G}$ with polar divisor $\mathcal{D}$,

such that $\left(f^{*} \mathcal{E}_{G}, f^{*} \nabla\right)=\left(E_{G}, \delta\right)$.

Let us recall a few elements of the proof of this (classical) result. Choose a covering $\mathfrak{U}$ of $X_{0} \backslash D_{0}$ by complex discs and a small neighborhood $U_{i}$ for each $x_{i} \in D_{0}$. Since $\delta$ is integrable, we can choose local charts for $E_{G}$ over $\mathfrak{U}$ such that all transition functions are constants. Now if the curve fits into an analytic family $\mathcal{X} \longrightarrow \mathcal{B}$, one can, restricting $\mathcal{B}$ if necessary, cover $\mathcal{X}$ by open subsets of the form $V_{j} \times B$, where $V_{j}$ are the open subsets of $X_{0}$ in the collection $\mathfrak{U} \cup\left\{U_{i}\right\}_{i=1}^{n}$. The isomonodromic deformation is then given by simply extending the transition maps by keeping them to be constant in deformation parameters.

The logarithmic connection $\delta$ gives a splitting of the logarithmic Atiyah bundle

$$
\operatorname{At}\left(E_{G}, D_{0}\right)=\operatorname{ad}\left(E_{G}\right) \oplus \mathrm{T} X_{0}\left(-D_{0}\right) .
$$

The corresponding cohomological decomposition

$$
\mathrm{H}^{1}\left(X_{0}, \operatorname{At}\left(E_{G}, D_{0}\right)\right)=\mathrm{H}^{1}\left(X_{0}, \operatorname{ad}\left(E_{G}\right)\right) \oplus \mathrm{H}^{1}\left(X_{0}, \mathrm{~T} X_{0}\left(-D_{0}\right)\right)
$$

gives a splitting of the infinitesimal deformations of $\left(X_{0}, D_{0}, E_{G}\right)$ into the infinitesimal deformations of $\left(X_{0}, D_{0}\right)$ and the infinitesimal deformations of $E_{G}$ (keeping $\left(X_{0}, D_{0}\right)$ fixed). In other words, let

$$
\delta^{\prime}: \mathrm{H}^{1}\left(X_{0}, \mathrm{~T} X_{0}\left(-D_{0}\right)\right) \longrightarrow \mathrm{H}^{1}\left(X_{0}, \operatorname{At}\left(E_{G}, D_{0}\right)\right)
$$

be the homomorphism induced by the homomorphism

$$
\delta: \mathrm{T} X_{0}\left(-D_{0}\right) \longrightarrow \operatorname{At}\left(E_{G}, D_{0}\right)
$$

in Lemma 4.1 defining the logarithmic connection on $E_{G}$. Given an infinitesimal deformation $(\mathcal{X}, q, \mathcal{D}, f)$ of $\left(X_{0}, D_{0}\right)$, the above homomorphism $\delta^{\prime}$ produces an infinitesimal deformation 
$\left(\mathcal{X}, q, \mathcal{D}, f, \mathcal{E}_{G}, \psi\right)$ of $\left(X_{0}, D_{0}, E_{G}\right)$. As explained above, this holomorphic principal $G$ bundle $\mathcal{E}_{G}$ on $\mathcal{X}$ coincides with the holomorphic principal $G$-bundle on $\mathcal{X}$ produced by the isomonodromic deformation in Lemma 4.1.

We will now construct the exact sequence in (2.11) corresponding to the above infinitesimal deformation $\left(\mathcal{X}, q, \mathcal{D}, f, \mathcal{E}_{G}, \psi\right)$. Consider the injective homomorphism

$$
\mathrm{T} X_{0}\left(-D_{0}\right) \longrightarrow \operatorname{At}\left(E_{G}, D_{0}\right) \oplus f^{*} \mathrm{~T} \mathcal{X}(-\mathcal{D}), \quad v \longmapsto(\delta(v),-(\mathrm{d} f)(v)),
$$

where $\mathrm{d} f$ is the differential in (2.2) and $\delta$ is the homomorphism in (4.3). The corresponding cokernel

$$
\operatorname{At}^{\delta}\left(E_{G}, D_{0}\right):=\left(\operatorname{At}\left(E_{G}, D_{0}\right) \oplus f^{*} \mathrm{~T} \mathcal{X}(-\mathcal{D})\right) /\left(\mathrm{T} X_{0}\left(-D_{0}\right)\right)
$$

possesses a canonical projection

$$
\widehat{\delta}: \operatorname{At}^{\delta}\left(E_{G}, D_{0}\right) \longrightarrow \mathcal{O}_{X_{0}}, \quad(v, w) \longmapsto h(w),
$$

where $h$ is the homomorphism in (2.2); note that the above homomorphism $\widehat{\delta}$ is well-defined because $h$ vanishes on the image of $\mathrm{T}_{0}\left(-D_{0}\right)$ in $\operatorname{At}\left(E_{G}, D_{0}\right) \oplus f^{*} \mathrm{~T} \mathcal{X}(-\mathcal{D})$. The kernel of $\widehat{\delta}$ is identified with $\operatorname{At}\left(E_{G}, D_{0}\right)$ by sending any $z \in \operatorname{At}\left(E_{G}, D_{0}\right)$ to the image in $\operatorname{At}^{\delta}\left(E_{G}, D_{0}\right)$ of $(z, 0) \in \operatorname{At}\left(E_{G}, D_{0}\right) \oplus f^{*} \mathrm{~T} \mathcal{X}(-\mathcal{D})$. Therefore, we obtain the following exact sequence of vector bundles over $X_{0}$ :

$$
0 \longrightarrow \operatorname{At}\left(E_{G}, D_{0}\right) \longrightarrow \operatorname{At}^{\delta}\left(E_{G}, D_{0}\right) \stackrel{\widehat{\delta}}{\longrightarrow} \mathcal{O}_{X_{0}} \longrightarrow 0
$$

This exact sequence coincides with the one in (2.11). In particular, we have $\operatorname{At}^{\delta}\left(E_{G}, D_{0}\right)=$ $f^{*} \operatorname{At}\left(\mathcal{E}_{G}\right)$.

Consider the projection

$$
\operatorname{At}\left(E_{G}, D_{0}\right) \oplus f^{*} \mathrm{~T} \mathcal{X}(-\mathcal{D}) \longrightarrow \operatorname{ad}\left(E_{G}\right), \quad\left(z_{1}, z_{2}\right) \longmapsto \delta^{\prime \prime}\left(z_{1}\right),
$$

where $\delta^{\prime \prime}$ is constructed in (4.2) from $\delta$. It vanishes on the image of $\mathrm{T} X_{0}\left(-D_{0}\right)$, yielding a projection

$$
\lambda: \operatorname{At}^{\delta}\left(E_{G}, D_{0}\right) \longrightarrow \operatorname{ad}\left(E_{G}\right) .
$$

Let $\nabla^{\prime \prime}: \operatorname{At}\left(\mathcal{E}_{G}, \mathcal{D}\right) \longrightarrow \operatorname{ad}\left(\mathcal{E}_{G}\right)$ be the homomorphism given by the logarithmic connection $\nabla$ in Lemma 4.1, The homomorphism in (4.5) fits in the commutative diagram

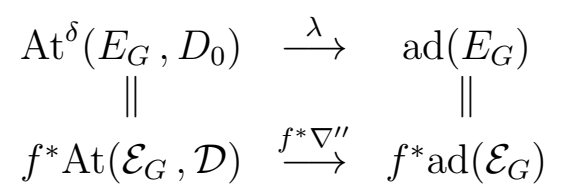

(the vertical identifications are evident).

We summarize the above constructions in the following lemma:

Lemma 4.2. Given $(\mathcal{X}, q, \mathcal{D}, f)$ as in (2.1), and also given a logarithmic connection $\delta$ on a holomorphic principal $G$-bundle $E_{G} \longrightarrow X_{0}$, the exact sequence in (2.11) corresponding to the infinitesimal deformation of $\left(X_{0}, D_{0}, E_{G}\right)$ in Lemma 4.1 is

$$
0 \longrightarrow \operatorname{At}\left(E_{G}, D_{0}\right) \longrightarrow \operatorname{At}^{\delta}\left(E_{G}, D_{0}\right) \stackrel{\widehat{\delta}}{\longrightarrow} \mathcal{O}_{X_{0}} \longrightarrow 0
$$

where $\widehat{\delta}$ is constructed in (4.4). 
4.2. The second fundamental form. Fix a logarithmic connection $\delta$ on $\left(E_{G}, D_{0}\right)$ as in (4.1). Take a holomorphic reduction of structure group $E_{P} \subset E_{G}$ to $P$ as in (3.1). The composition

$$
S(\delta):=\mu \circ \delta: \mathrm{T} X_{0}\left(-D_{0}\right) \longrightarrow E_{P}(\mathfrak{g} / \mathfrak{p})
$$

where $\mu$ is constructed in (3.2), is called the second fundamental form of $E_{P}$ for the connection $\delta$. We note that $\delta$ is induced by a logarithmic connection on the holomorphic principal $P$-bundle $E_{P}$ if and only if we have $S(\delta)=0$.

Assume that $E_{P}$ satisfies the condition that $S(\delta) \neq 0$. Let

$$
\mathcal{L} \subset E_{P}(\mathfrak{g} / \mathfrak{p})
$$

be the holomorphic line subbundle generated by the image of the homomorphism $S(\delta)$ in (4.7). More precisely, $\mathcal{L}$ is the inverse image, in $E_{P}(\mathfrak{g} / \mathfrak{p})$, of the torsion part of the quotient $E_{P}(\mathfrak{g} / \mathfrak{p}) /\left(S(\delta)\left(\mathrm{T} X_{0}\left(-D_{0}\right)\right)\right)$. Now consider the homomorphism

$$
S(\delta)^{\prime}: \mathrm{T} X_{0}\left(-D_{0}\right) \longrightarrow \mathcal{L}
$$

given by the second fundamental form $S(\delta)$. Let

$$
S^{\prime}: \mathrm{H}^{1}\left(X_{0}, \mathrm{~T} X_{0}\left(-D_{0}\right)\right) \longrightarrow \mathrm{H}^{1}\left(X_{0}, \mathcal{L}\right)
$$

be the homomorphism of cohomologies induced by $S(\delta)^{\prime}$ in (4.9).

Proposition 4.3. As before, let $\delta$ be a logarithmic connection on $E_{G} \longrightarrow X_{0}$ with polar divisor $D_{0}$, and let $(\mathcal{X}, q, \mathcal{D}, f)$ be an infinitesimal deformation of $\left(X_{0}, D_{0}\right)$. Let $\mathcal{E}_{G} \longrightarrow \mathcal{X}$ be the isomonodromic deformation of $\delta$ along $(\mathcal{X}, \mathcal{D})$ obtained in Lemma 4.1. Let $E_{P} \subset E_{G}$ be a holomorphic reduction of structure group to $P$ over $X_{0}$ that extends to a holomorphic reduction of structure group $\mathcal{E}_{P} \subset \mathcal{E}_{G}$ to $P$ over $\mathcal{X}$. Then

$$
S^{\prime}\left(\phi\left(1_{X_{0}}\right)\right)=0,
$$

where $\phi\left(1_{X_{0}}\right)$ is the cohomology class constructed in (2.4) corresponding to $(\mathcal{X}, q, \mathcal{D}, f)$, and $S^{\prime}$ is constructed in (4.10).

Proof. Consider the inverse images

$$
\operatorname{At}_{P}\left(E_{G}, D_{0}\right):=\mu^{-1}(\mathcal{L}) \subset \operatorname{At}\left(E_{G}, D_{0}\right) \text { and } \operatorname{ad}_{P}\left(E_{G}\right):=\mu_{1}^{-1}(\mathcal{L}) \subset \operatorname{ad}\left(E_{G}\right),
$$

where $\mu$ and $\mu_{1}$ are the quotient maps in (3.2), and $\mathcal{L}$ is constructed in (4.8). These two vector bundles fit in the following commutative diagram produced from (3.2):

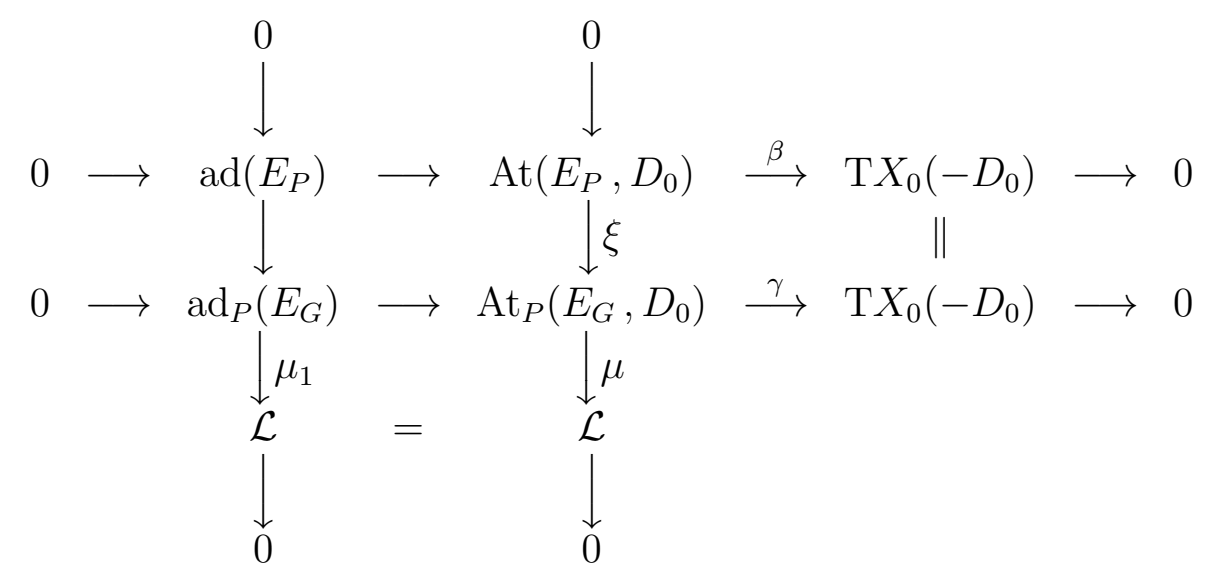


By the construction of $\operatorname{At}_{P}\left(E_{G}, D_{0}\right)$, the connection homomorphism $\delta$ in (4.3) factors through a homomorphism

$$
\delta^{1}: \mathrm{T} X_{0}\left(-D_{0}\right) \longrightarrow \operatorname{At}_{P}\left(E_{G}, D_{0}\right)
$$

Consider the homomorphism

$$
\delta_{*}^{1}: \mathrm{H}^{1}\left(X_{0}, \mathrm{~T} X_{0}\left(-D_{0}\right)\right) \longrightarrow \mathrm{H}^{1}\left(X_{0}, \operatorname{At}_{P}\left(E_{G}, D_{0}\right)\right)
$$

induced by the above homomorphism $\delta^{1}$, and let

$$
\Phi:=\delta_{*}^{1}\left(\phi\left(1_{X_{0}}\right)\right) \in \mathrm{H}^{1}\left(X_{0}, \operatorname{At}_{P}\left(E_{G}, D_{0}\right)\right)
$$

be the image of the cohomology class $\phi\left(1_{X_{0}}\right)$ that characterizes the deformation $(\mathcal{X}, \mathcal{D})$ as in (2.4).

As in the statement of the proposition, let $\mathcal{E}_{P} \longrightarrow \mathcal{X}$ be a holomorphic extension of the reduction $E_{P}$. Note that from (3.6), (3.2) and Lemma 4.2 we have

$$
\begin{gathered}
\operatorname{At}\left(E_{G}, D_{0}\right) / \operatorname{At}\left(E_{P}, D_{0}\right)=E_{P}(\mathfrak{g} / \mathfrak{p})=\left(f^{*} \operatorname{At}\left(\mathcal{E}_{G}, \mathcal{D}\right)\right) /\left(f^{*} \operatorname{At}\left(\mathcal{E}_{P}, \mathcal{D}\right)\right) \\
=\operatorname{At}^{\delta}\left(E_{G}, D_{0}\right) / f^{*} \operatorname{At}\left(\mathcal{E}_{P}, \mathcal{D}\right)
\end{gathered}
$$

Let $\mu_{2}: \operatorname{At}^{\delta}\left(E_{G}, D_{0}\right) \longrightarrow E_{P}(\mathfrak{g} / \mathfrak{p})$ be the above quotient map. Define

$$
\operatorname{At}_{P}^{\delta}\left(E_{G}, D_{0}\right):=\mu_{2}^{-1}(\mathcal{L}) \subset \operatorname{At}^{\delta}\left(E_{G}, D_{0}\right)
$$

where $\mathcal{L}$ is constructed in (4.8).

Now we have the following commutative diagram:

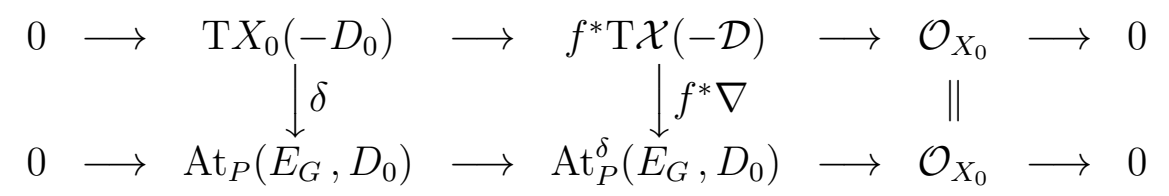

where the bottom exact sequence is obtained from (3.6), and the top exact sequence is as in (2.2) (see also (4.6) $)$. Let

$$
\nu: \mathrm{H}^{0}\left(X_{0}, \mathcal{O}_{X_{0}}\right) \longrightarrow \mathrm{H}^{1}\left(X_{0}, \operatorname{At}_{P}\left(E_{G}, D_{0}\right)\right)
$$

be the connecting homomorphism in the long exact sequence of cohomologies associated to the bottom exact sequence in (4.14). From the commutativity of (4.14) and the construction of $\phi\left(1_{X_{0}}\right)$ (see (2.4) ) it follows that

$$
\nu\left(1_{X_{0}}\right)=\delta_{*}^{1}\left(\phi\left(1_{X_{0}}\right)\right)=\Phi,
$$

where $\nu$ and $\delta_{*}^{1}$ are the homomorphisms constructed in (4.15) and (4.12) respectively, and $\Phi$ is the cohomology class in (4.13). 
The diagram in (3.6) produces the diagram

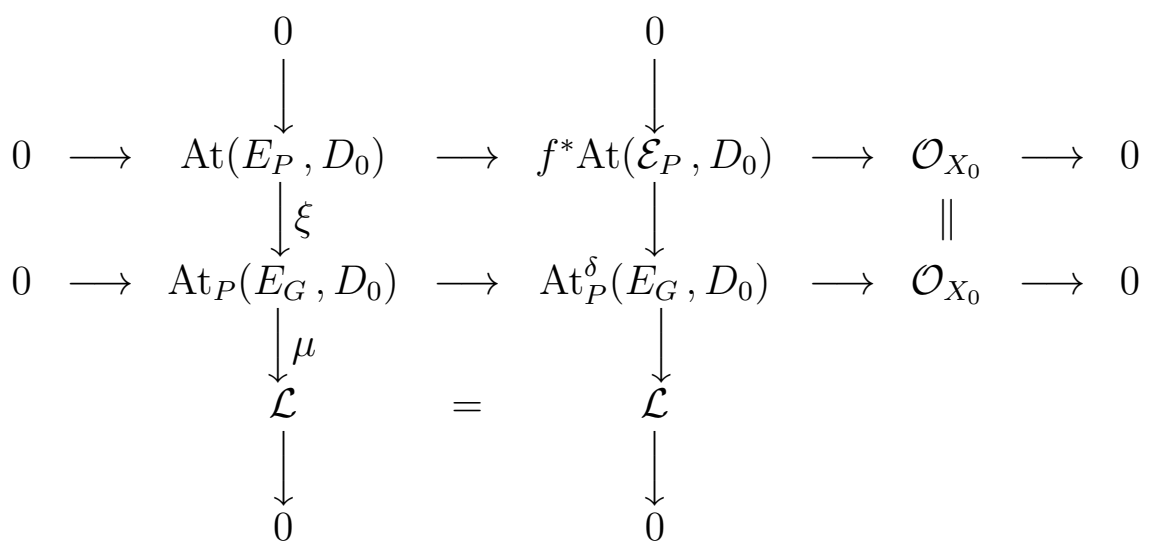

where $\xi$ and $\mu$ are the homomorphisms in (4.11). Using this diagram we can check that

$$
\Phi=\xi_{*}(\theta),
$$

where $\theta$ is the cohomology classes in (3.5), and

$$
\xi_{*}: \mathrm{H}^{1}\left(X_{0}, \operatorname{At}\left(E_{P}, D_{0}\right)\right) \longrightarrow \mathrm{H}^{1}\left(X_{0}, \operatorname{At}_{P}\left(E_{G}, D_{0}\right)\right)
$$

is the homomorphism induced by $\xi$ in (4.17). Indeed, (4.18) follows from (4.16), the commutativity of (4.17) and the construction of $\theta$.

Let $\mu_{*}: \mathrm{H}^{1}\left(X_{0}, \operatorname{At}_{P}\left(E_{G}, D_{0}\right)\right) \longrightarrow \mathrm{H}^{1}\left(X_{0}, \mathcal{L}\right)$ be the homomorphism induced by the homomorphism $\mu$ in (4.17). It is straight-forward to check that

$$
\mu_{*}(\Phi)=S^{\prime}\left(\phi\left(1_{X_{0}}\right)\right)
$$

(see (4.10), (2.4) and (4.13) for $S^{\prime}, \phi\left(1_{X_{0}}\right)$ and $\Phi$ respectively). Indeed, this follows from (4.16) and the definition of $S(\delta)$ in (4.7). Combining this with (4.18), we have

$$
S^{\prime}\left(\phi\left(1_{X_{0}}\right)\right)=\mu_{*}(\Phi)=\mu_{*}\left(\xi_{*}(\theta)\right) .
$$

Since $\mu \circ \xi=0$ (see (4.17) $)$, it now follows that $S^{\prime}\left(\phi\left(1_{X_{0}}\right)\right)=0$.

\section{LOGARITHMIC CONNECTIONS AND SEMISTABILITY OF THE UNDERLYING PRINCIPAL BUNDLE}

Let $\mathcal{T}_{g, n}$ be the Teichmüller space of genus $g$ compact Riemann surfaces with $n$ ordered marked points. As before, we assume that $g>0$, and if $g=1$, then $n>0$. Let

$$
\tau: \mathcal{C} \longrightarrow \mathcal{T}_{g, n}
$$

be the universal Teichmüller curve with $n$ ordered sections $\Sigma$. The fiber of $\mathcal{C}$ over any point $t \in \mathcal{T}_{g, n}$ will be denoted by $\mathcal{C}_{t}$. The ordered subset $\mathcal{C}_{t} \cap \Sigma \subset \mathcal{C}_{t}$ will be denoted by $\Sigma_{t}$.

Take a $n$-pointed Riemann surface $\left(C_{0}, \Sigma_{0}\right)$ of genus $g$, which is represented by a point of $\mathcal{T}_{g, n}$. Let

$$
\nabla_{0}
$$

be a logarithmic connection on a holomorphic principal $G$-bundle $F_{G} \longrightarrow C_{0}$ with polar divisor $\Sigma_{0}$. By the Riemann-Hilbert correspondence, the connection $\nabla_{0}$ produces a flat 
(isomonodromic) logarithmic connection $\nabla$ on a holomorphic principal $G$-bundle $\mathcal{F}_{G} \longrightarrow \mathcal{C}$ with polar divisor $\Sigma$.

The following lemma is a special case of the main theorem in GN] (see also [Sh]). Although the families of $G$-bundles considered in [GN] are algebraic, all arguments there go through in the analytic case of our interest with obvious modifications.

Lemma $5.1([\mathrm{GN}])$. Let $\mathcal{F}_{G} \longrightarrow \mathcal{C} \longrightarrow \mathcal{T}_{g, n}$ be as above. For each Harder-Narasimhan type $\kappa$, the set

$$
\mathcal{Y}_{\kappa}:=\left\{t \in \mathcal{T}_{g, n}\left|\mathcal{F}_{G}\right|_{\mathcal{C}_{t}} \text { is of type } \kappa\right\}
$$

is a (possibly empty) locally closed complex analytic subspace of $\mathcal{T}_{g, n}$. More precisely, for each Harder-Narasimhan type $\kappa$, the union $\mathcal{Y}_{\leq \kappa}:=\bigcup_{\kappa^{\prime} \leq \kappa} \mathcal{Y}_{\kappa^{\prime}}$ is a closed complex analytic subset of $\mathcal{T}_{g, n}$. Moreover, the principal $G$-bundle

$$
\left.\mathcal{F}_{G}\right|_{\tau^{-1}\left(\mathcal{Y}_{\kappa}\right)} \longrightarrow \tau^{-1}\left(\mathcal{Y}_{\kappa}\right)
$$

possesses a canonical holomorphic reduction of structure group inducing the Harder-Narasimhan reduction of $\left.\mathcal{F}_{G}\right|_{\mathcal{C}_{t}}$ for every $t \in \mathcal{Y}_{\kappa}$.

In the following two Sections 5.1 and 5.2, we will see that under certain assumptions, the only Harder-Narasimhan stratum $\mathcal{Y}_{\kappa}$ of maximal dimension $\operatorname{dim}\left(\mathcal{T}_{g, n}\right)=3 g-3+n$ is the trivial one, in the sense that the Harder-Narasimhan parabolic subgroup is $G$ itself. In other words, if the principal $G$-bundle $F_{G}$ is not semistable, and therefore has a non-trivial Harder-Narasimhan reduction $E_{P} \subset E_{G}$ to a certain parabolic subgroup $P \subsetneq G$, then there is always an isomonodromic deformation in which direction the reduction $E_{P}$ is obstructed meaning it does not extend.

5.1. The case of $n=0$. In this subsection we assume that $n=0$. So, we have $g>1$.

Theorem 5.2. There is a closed complex analytic subset $\mathcal{Y} \subset \mathcal{T}_{g, 0}$ of codimension at least $g$ such that for every $t \in \mathcal{T}_{g, 0} \backslash \mathcal{Y}$, the holomorphic principal $G$-bundle $\left.\mathcal{F}_{G}\right|_{\mathcal{C}_{t}} \longrightarrow \mathcal{C}_{t}$ is semistable.

Proof. Let $\mathcal{Y} \subset \mathcal{T}_{g, 0}$ denote the (finite) union of all Harder-Narasimhan strata $\mathcal{Y}_{\kappa}$ as in Lemma 5.1 with nontrivial Harder-Narasimhan type $\kappa$. From Lemma 5.1 we know that $\mathcal{Y}$ is a closed complex analytic subset of $\mathcal{T}_{g, 0}$.

Take any $t \in \mathcal{Y}_{\kappa} \subset \mathcal{Y}$. Let $E_{G}=\left.\mathcal{F}_{G}\right|_{\mathcal{C}_{t}}$ be the holomorphic principal $G$-bundle on $X_{0}:=\mathcal{C}_{t}$. The holomorphic connection on $E_{G}$ obtained by restricting $\nabla$ will be denoted by $\delta$. Since $E_{G}$ is not semistable, there is a proper parabolic subgroup $P \subsetneq G$ and a holomorphic reduction of structure group $E_{P} \subset E_{G}$ to $P$, such that $E_{P}$ is the HarderNarasimhan reduction [Be], $\mathrm{AAB}$; the type of this Harder-Narasimhan reduction is $\kappa$. From Lemma 5.1 we know that $E_{P}$ extends to a holomorphic reduction of structure group of the principal $G$-bundle $\left.\mathcal{F}_{G}\right|_{\tau^{-1}\left(\mathcal{Y}_{\kappa}\right)}$ to the subgroup $P$.

Let $\operatorname{ad}\left(E_{P}\right)$ and $\operatorname{ad}\left(E_{G}\right)$ be the adjoint vector bundles of $E_{P}$ and $E_{G}$ respectively. Consider the vector bundle

$$
\operatorname{ad}\left(E_{G}\right) / \operatorname{ad}\left(E_{P}\right)=E_{P}(\mathfrak{g} / \mathfrak{p})
$$


$($ see $(3.2))$. We know that

$$
\mu_{\max }\left(E_{P}(\mathfrak{g} / \mathfrak{p})\right)<0
$$

AAB, p. 705] (see sixth line from bottom). In particular

$$
\operatorname{degree}\left(E_{P}(\mathfrak{g} / \mathfrak{p})\right)<0 .
$$

A holomorphic connection on $E_{G}$ induces a holomorphic connection on $\operatorname{ad}\left(E_{G}\right)$, hence degree $\left(\operatorname{ad}\left(E_{G}\right)\right)=0$. Combining this with (5.3) it follows that degree $\left(\operatorname{ad}\left(E_{P}\right)\right)>0$, because $\operatorname{ad}\left(E_{G}\right) / \operatorname{ad}\left(E_{P}\right)=E_{P}(\mathfrak{g} / \mathfrak{p})$. Since degree $\left(\operatorname{ad}\left(E_{P}\right)\right) \neq 0$, the holomorphic vector bundle $\operatorname{ad}\left(E_{P}\right)$ does not admit any holomorphic connection, hence the principal $P$-bundle $E_{P}$ does not admit a holomorphic connection. Consequently, the second fundamental form $S(\delta)$ of $E_{P}$ for $\delta$ (see (4.7)) is nonzero.

Using the second fundamental form $S(\delta)$, construct the holomorphic line subbundle

$$
\mathcal{L} \subset E_{P}(\mathfrak{g} / \mathfrak{p})
$$

as done in (4.8). From (5.2) we have

$$
\operatorname{degree}(\mathcal{L})<0
$$

Consider the short exact sequence of sheaves on $X_{0}$

$$
0 \longrightarrow \mathrm{T} X_{0} \stackrel{S(\delta)^{\prime}}{\longrightarrow} \mathcal{L} \longrightarrow T^{\delta}:=\mathcal{L} /\left(S(\delta)\left(\mathrm{T} X_{0}\right)\right) \longrightarrow 0
$$

where $S(\delta)^{\prime}$ is constructed in (4.9); note that $T^{\delta}$ is a torsion sheaf because $S(\delta)^{\prime} \neq 0$ (recall that $S(\delta) \neq 0$ ). From (5.4) it follows that

$$
\operatorname{degree}\left(T^{\delta}\right)=\operatorname{degree}(\mathcal{L})-\operatorname{degree}\left(\mathrm{T} X_{0}\right)<-\operatorname{degree}\left(\mathrm{T} X_{0}\right)=2 g-2 .
$$

So, we have

$$
\operatorname{dim} \mathrm{H}^{0}\left(X_{0}, T^{\delta}\right)=\operatorname{degree}\left(T^{\delta}\right)<2 g-2=\operatorname{dim} \mathrm{H}^{1}\left(X_{0}, \mathrm{~T} X_{0}\right)+1-g .
$$

This implies that

$$
\operatorname{dim} \mathrm{H}^{1}\left(X_{0}, \mathrm{~T} X_{0}\right)-\operatorname{dim} \mathrm{H}^{0}\left(X_{0}, T^{\delta}\right) \geq g .
$$

Consider the long exact sequence of cohomologies

$$
\mathrm{H}^{0}\left(X_{0}, T^{\delta}\right) \longrightarrow \mathrm{H}^{1}\left(X_{0}, \mathrm{~T} X_{0}\right) \stackrel{\zeta}{\longrightarrow} \mathrm{H}^{1}\left(X_{0}, \mathcal{L}\right)
$$

associated to the short exact sequence of sheaves in (5.5). From (5.6) it follows that

$$
\operatorname{dim} \zeta\left(\mathrm{H}^{1}\left(X_{0}, \mathrm{~T} X_{0}\right)\right) \geq g
$$

Since the reduction $E_{P}$ extends to a holomorphic reduction of structure group of the principal $G$-bundle $\left.\mathcal{F}_{G}\right|_{\tau^{-1}\left(\mathcal{Y}_{\kappa}\right)}$ to the subgroup $P$, combining (5.7) and Proposition 4.3 we conclude that the codimension of the complex analytic subset $\mathcal{Y}_{\kappa} \subset \mathcal{T}_{g, 0}$ is at least $g$. This completes the proof of the theorem. 
5.2. When $n$ is arbitrary. Now we assume that $n>0$.

A logarithmic connection $\eta$ on a holomorphic principal $G$-bundle $F_{G} \longrightarrow X_{0}$ is called reducible if there is pair $\left(P, F_{P}\right)$, where $P \subsetneq G$ is a parabolic subgroup and $F_{P} \subset F_{G}$ is a holomorphic reduction of structure group of $F_{G}$ to $P$, such that $\eta$ is induced by a connection on $F_{P}$. Note that $\eta$ is induced by a connection on $F_{P}$ if and only if the second fundamental form of $F_{P}$ for $\eta$ vanishes identically. A connection is called irreducible if it is not reducible or, equivalently, if the monodromy representation of the corresponding flat principal $G$-bundle does not factor through any proper parabolic subgroup of $G$.

Proposition 5.3. Assume that the logarithmic connection $\nabla_{0}$ in (5.1) is irreducible. Then there is a closed complex analytic subset $\mathcal{Y} \subset \mathcal{T}_{g, n}$ of codimension at least $g$ such that for every $t \in \mathcal{T}_{g, n} \backslash \mathcal{Y}$, the holomorphic principal $G$-bundle $\left.\mathcal{F}_{G}\right|_{\mathcal{C}_{t}}$ is semistable.

Proof. The proof of Theorem 5.2 goes through after some obvious modifications. As before, let $\mathcal{Y} \subset \mathcal{T}_{g, n}$ be the locus of all points $t$ such that the principal $G$-bundle $\left.\mathcal{F}_{G}\right|_{\mathcal{C}_{t}}$ is not semistable. Take any point $t \in \mathcal{Y}_{\kappa} \subset \mathcal{Y}$. Let $E_{G}=\left.\mathcal{F}_{G}\right|_{\mathcal{C}_{t}}$ be the holomorphic principal $G$-bundle on $X_{0}:=\mathcal{C}_{t}$. The logarithmic connection on $E_{G}$ with polar divisor $D_{0}:=\Sigma_{t}$ obtained by restricting $\nabla$ will be denoted by $\delta$.

Let $E_{P} \subset E_{G}$ be the Harder-Narasimhan reduction; its type is $\kappa$. Since $\nabla_{0}$ is irreducible, the second fundamental form $S(\delta)$ of $E_{P}$ for $\delta$ (see (4.77) ) is nonzero. We note that for the monodromy of a logarithmic connection, the property of being irreducible is preserved under isomonodromic deformations. Consider the short exact sequence of sheaves on $X_{0}$

$$
0 \longrightarrow \mathrm{T} X_{0}\left(-D_{0}\right) \stackrel{S(\delta)^{\prime}}{\longrightarrow} \mathcal{L} \longrightarrow T^{\delta}:=\mathcal{L} /\left(S(\delta)\left(\mathrm{T} X_{0}\left(-D_{0}\right)\right)\right) \longrightarrow 0,
$$

where $S(\delta)^{\prime}$ is constructed in (4.9). As before, $\operatorname{degree}(\mathcal{L})<0$, because $\mu_{\max }\left(E_{P}(\mathfrak{g} / \mathfrak{p})\right)<0$. So,

$$
\operatorname{degree}\left(T^{\delta}\right)=\operatorname{degree}(\mathcal{L})-\operatorname{degree}\left(\mathrm{T} X_{0}\left(-D_{0}\right)\right)<-\operatorname{degree}\left(\mathrm{T} X_{0}\left(-D_{0}\right)\right)=2 g-2+n .
$$

We now have

$$
\operatorname{dim} \mathrm{H}^{0}\left(X_{0}, T^{\delta}\right)=\operatorname{degree}\left(T^{\delta}\right)<2 g-2+n=\operatorname{dim} \mathrm{H}^{1}\left(X_{0}, \mathrm{~T} X_{0}\left(-D_{0}\right)\right)+1-g .
$$

Hence the dimension of the image of the homomorphism

$$
\mathrm{H}^{1}\left(X_{0}, \mathrm{~T} X_{0}\left(-D_{0}\right)\right) \longrightarrow \mathrm{H}^{1}\left(X_{0}, \mathcal{L}\right)
$$

in the long exact sequence of cohomologies associated to (5.8) is at least $g$. Since the reduction $E_{P}$ extends to a holomorphic reduction of $\left.\mathcal{F}_{G}\right|_{\tau^{-1}\left(\mathcal{Y}_{\kappa}\right)}$ to $P$, and the dimension of the image of the homomorphism in (5.9) is at least $g$, from Proposition 4.3 we conclude that the codimension of $\mathcal{Y}_{\kappa} \subset \mathcal{T}_{g, 0}$ is at least $g$.

\subsection{Stability of underlying principal bundle. We now assume that $g \geq 2$.}

Proposition 5.4. Assume that the logarithmic connection $\nabla_{0}$ in (5.1) is irreducible. There is a closed analytic subset $\mathcal{Y}^{\prime} \subset \mathcal{T}$ of codimension at least $g-1$ such that for every $t \in \mathcal{T}_{g} \backslash \mathcal{Y}$, the holomorphic principal $G$-bundle $\left.\mathcal{F}_{G}\right|_{\mathcal{C}_{t}}$ is stable. 
Proof. The proof is identical to the proof of Proposition 5.3. If $E_{G}=\left.\mathcal{F}_{G}\right|_{\mathcal{C}_{t}}$ is not stable, there is a maximal parabolic subgroup $P \subsetneq G$ and a holomorphic reduction of structure group $E_{P} \subset E_{G}$ to $P$, such that the quotient bundle

$$
\operatorname{ad}\left(E_{G}\right) / \operatorname{ad}\left(E_{P}\right)=E_{P}(\mathfrak{g} / \mathfrak{p})
$$

is semistable of degree zero. Therefore, we have degree $(\mathcal{L}) \leq 0$. This implies that

$$
\operatorname{degree}\left(T^{\delta}\right) \leq 2 g-2-n \text {. }
$$

Hence the dimension of the image of the homomorphism $\mathrm{H}^{1}\left(X_{0}, \mathrm{~T} X_{0}\left(-D_{0}\right)\right) \longrightarrow \mathrm{H}^{1}\left(X_{0}, \mathcal{L}\right)$ in the long exact sequence of cohomologies associated to (5.8) is at least $g-1$.

\section{ACKNOWLEDGEMENTS}

We thank the referee for helpful comments. We thank Université de Brest for hospitality where the work was initiated. The first author is supported by a J. C. Bose Fellowship. The second author is supported by ANR-13-BS01-0001-01 and ANR-13-JS01-0002-01.

\section{REFERENCES}

[AAB] B. Anchouche, H. Azad and I. Biswas, Harder-Narasimhan reduction for principal bundles over a compact Kähler manifold, Math. Ann. 323 (2002), 693-712.

[AB] D. Anosov and A. Bolibruch, The Riemann-Hilbert problem, Aspects of Mathematics, E22. Friedr. Vieweg \& Sohn, Braunschweig, 1994.

[At] M. F. Atiyah, Complex analytic connections in fibre bundles, Trans. Amer. Math. Soc. 85 (1957), $181-207$.

[Be] K. A. Behrend, Semistability of reductive group schemes over curves, Math. Ann. 301 (1995), 281305 .

[Boa] P. Boalch, G-bundles, isomonodromy and quantum Weyl groups, Int. Math. Res. Not. 22 (2002), 1129-1166

[Bol1] A. Bolibruch, On sufficient conditions for the positive solvability of the Riemann-Hilbert problem, Mathem. Notes of the Acad. Sci. USSR 51 (1992), 110-117.

[Bol2] A. Bolibruch, The Riemann-Hilbert problem, Russian Math. Surveys 45 (1990), 1-58.

[De] W. Dekkers, The matrix of a connection having regular singularities on a vector bundle of rank 2 on $\mathbb{P}^{1}(\mathbb{C})$, Équations différentielles et systèmes de Pfaff dans le champ complexe (Sem., Inst. Rech. Math. Avancée, Strasbourg, 1975), pp. 33-43, Lecture Notes in Math., 712, Springer, Berlin, 1979.

[EH] H. Esnault and C. Hertling, Semistable bundles and reducible representations of the fundamental group, Int. Jour. Math. 12 (2001), 847-855.

[EV] H. Esnault and E. Viehweg, Semistable bundles on curves and irreducible representations of the fundamental group, Algebraic geometry: Hirzebruch 70 (Warsaw, 1998), 129-138, Contemp. Math., 241, Amer. Math. Soc., Providence, RI, 1999.

[GN] S. R. Gurjar and N. Nitsure, Schematic Harder-Narasimhan stratification for families of principal bundles and lambda modules, Proc. Ind. Acad. Sci. (Math. Sci.) 124 (2014), 315-332.

[He1] V. Heu, Stability of rank 2 vector bundles along isomonodromic deformations, Math. Ann. 60 (2010), $515-549$.

[He2] V. Heu, Universal isomonodromic deformations of meromorphic rank 2 connections on curves, Ann. Inst. Fourier $\mathbf{3 4 4}$ (2009), 463-490.

[Ko] V. Kostov, Fuchsian linear systems on $\mathbb{C P}^{1}$ and the Riemann-Hilbert problem, Com. Ren. Acad. Sci. Paris 315 (1992), 143-148.

[Pl] J. Plemelj, Problems in the sense of Riemann and Klein, Interscience Tracts in Pure and Applied Mathematics, 16, Interscience Publishers John Wiley \& Sons Inc., New York-London-Sydney, 1964.

[Sa] C. Sabbah, Déformations isomonodromiques et variétés de Frobenius, Savoirs Actuels. Mathématiques. EDP Sciences, Les Ulis., CNRS Éditions, Paris, 2002. 
[Sh] S. S. Shatz, The decomposition and specialization of algebraic families of vector bundles, Compositio Math. 35 (1977), 163-187.

School of Mathematics, Tata Institute of Fundamental Research, Homi Bhabha Road, BOMBAY 400005, INDIA

E-mail address: indranil@math.tifr.res.in

Institut de Recherche Mathématique Avancée, 7 Rue René-Descartes, 67084 Strasbourg Cedex, France

E-mail address: heu@math.unistra.fr

Department of Mathematics, McGill University, Burnside Hall, 805 Sherbrooke St. W., Montreal, Que. H3A 0B9, Canada

E-mail address: jacques.hurtubise@mcgill.ca 